\title{
A STUDY OF AREA AND THICKNESS COMPRESSION OF PARAFFIN SECTIONS
}

\author{
YU XIANG, YANG GUO AND ZHENG-WEI YANG ${ }^{\circledR}$ \\ Morphometric Research Laboratory, North Sichuan Medical College, 234 Fujiang Road, Nanchong, Sichuan \\ 637000, China \\ e-mail: zwyang@nsmc.edu.cn \\ (Received December 3, 2017; revised August 21, 2018; accepted August 22, 2018)
}

\begin{abstract}
This study was carefully designed to determine the section compression of paraffin embedded sections. Two sections (one with thickness $5 \mu \mathrm{m}$ and one $10 \mu \mathrm{m}$ set by microtome) were cut from each of 2 sets of 12 testicular tissue (adult rats) blocks and stained with hematoxylin. Using scanned images, the area and the vertical (along the sectioning direction) and horizontal diameters of the block face were measured and compared with those of the unstained, stained or coverslipped section. Using the coverslipped section, the vertical and horizontal diameters of round spermatid nuclear profiles and the actual thickness of section were measured with light microscopy. Overall, the area of the coverslipped section was reduced by $5.5 \%-8.6 \%$ (on average) in comparison with that of the block face, with $69.5 \%-84.4 \%$ of the reduction being contributed by section compression in the process of section cutting, mounting and drying. The vertical (linear) compression of section, the primary cause of section area compression, was $5.9 \%-8.9 \%$. The vertical compression of nuclear profiles was $1.5 \%-2.3 \%$ in 2 sets of sections and $5.2 \%-5.7 \%$ in other sections, indicating a non-uniform compression of structures within some sections depending on procedures of section drying. The measured mean thickness of sections decreased by $3.1 \%-5.0 \%$.
\end{abstract}

Keywords: nuclear diameter, paraffin sections, section compression, section area, section thickness.

\section{INTRODUCTION}

Microscopic structures are generally observed on their sections, which, when used for stereological studies, are generally assumed to be the true 2-dimensional sectional images of the 3-dimensional structures without any geometric or topological changes. In practice, however, many changes - mainly tissue shrinkage and section compression in sum - occur with soft tissue sections, which may directly and substantially affect stereological or morphometric results one way or another (Dorph-Petersen et al., 2001; Yang, 2012). Incongruently, few studies specifically addressed the issues and possible resulting biases were rarely corrected. This study aimed to determine the area and thickness compression of paraffin-embedded biological tissue sections - commonly used in histology and often associated with marked morphological changes in comparison with resin-embedded sections (Yang, 2012), thus providing some methods and data for consideration or reference of future studies.

\section{MATERIALS AND METHODS}

Rats used in the present study for tissue samples were obtained from the Animal Center of North Sichuan Medical College. Experiment protocols were approved by the research section of the college and ethical guidelines constituted by the college were followed during experiment.

\section{FIRST EXPERIMENT}

Two testes, removed from a normal adult male SpragueDawley rat, were immersion-fixed in Bouin's solution for 2 days; 12 testicular slices, circular tissue blocks perpendicular to the testicular long axis and with thickness of approximately $2 \mathrm{~mm}$, were embedded in paraffin wax (Paraplast by Leica Biosystems Richmond, Illinois, USA; melting point $56^{\circ} \mathrm{C}$ ). The procedures prior to embedding were: absolute ethanol for $3+1 \mathrm{hrs}$ ( 3 hrs first in absolute ethanol and then 1 hour in fresh absolute ethanol), butanol for $1+2+2$ hrs, butanol plus paraffin (1:1) for $40 \mathrm{~min}$, and melted paraffin for $80+80 \mathrm{~min}$. 
With a semi-automatic microtome (RM2235, Leica Biosystems Nussloch GmbH, Nussloch, Germany) and a high profile microtome blade (818, Leica Biosystems Nussloch $\mathrm{GmbH}$ ), a couple of complete serial tissue sections (thickness set at 5 or $10 \mu \mathrm{m}$, alternatively chosen for different blocks) were first cut from each paraffin block, with the last section being obtained to minimize between- or within-section variation of thickness. A couple of hrs later, a few more serial sections (with a different thickness, 10 or $5 \mu \mathrm{m}$ ) were cut, with the last section being obtained again. Care was taken to ensure that the upper paraffin edge of the block was perpendicular to the direction of the cutting stroke. Immediately after either section was obtained, the section was floated onto an adhesive glass slide (with positively charged surface, produced by Yifan Experiment, China) from distilled water bath $\left(37^{\circ} \mathrm{C}\right)$ and then, after drying on hotplate at $37^{\circ} \mathrm{C}$ for a couple of hrs, scanned (resolution $2400 \mathrm{ppi}$ ) with a scanner (MRS-4800U2, Microtek, Shanghai, China) together with the block face of the tissue block left after the section was just cut (Fig. 1).

All sections were stained with periodic acid-Schiff's reagent and hematoxylin after a further drying on hotplate at $50^{\circ} \mathrm{C}$ for $2 \mathrm{hrs}$. The key staining procedures were: dewaxing in xylene (for $3 \times 2 \mathrm{~min}$ ); staining in $1 \%$ periodic acid (10 min), Schiff's reagent (10 min) and hematoxylin (5 min); dehydration in 70\% ethanol (2 $\mathrm{min}), 95 \%$ ethanol (2 min) and absolute ethanol $(2 \times 2 \mathrm{~min})$; clearing in xylene $(2 \times 2 \mathrm{~min})$; and cover- slipping with neutral balsam. The sections were scanned (as described above) first after clearing (and airdried) and then after coverslipping (and balsam solidified), respectively.

The vertical and horizontal diameters (Feret diameters) of the scanned image (JPEG file) of the block face (BF), unstained section (US, attached onto slide and not dewaxed), stained section (SS, after clearing) or coverslipped section (CS) were measured with an Adobe Photoshop 8.0.1 software. The image was first rotated so that its vertical direction (Y axis) represented the sectioning direction, which was determined with reference to the upper edge of the paraffin block and the edge of the glass slide. Then the Marquee tool was used to select and copy an arbitrary rectangle whose 2 parallel edges just passed through the upper and lower or the left and right end points of the image. The height or width of the rectangle, which could be directly read, was just the vertical or horizontal diameter of the image. (A ruler, which was additionally scanned with the image, was also measured to check the accuracy of the size measurement.)

The area of the scanned image was also measured with the Photoshop, by counting of test points - intersections between (software generated) vertical and horizontal lines superimposed on the image. The distance between neighboring parallel vertical or horizontal lines was set at $1.4 \mathrm{~mm}$. The area was estimated by multiplying the number of points hitting the image by the squared distance (Yang, 2012).
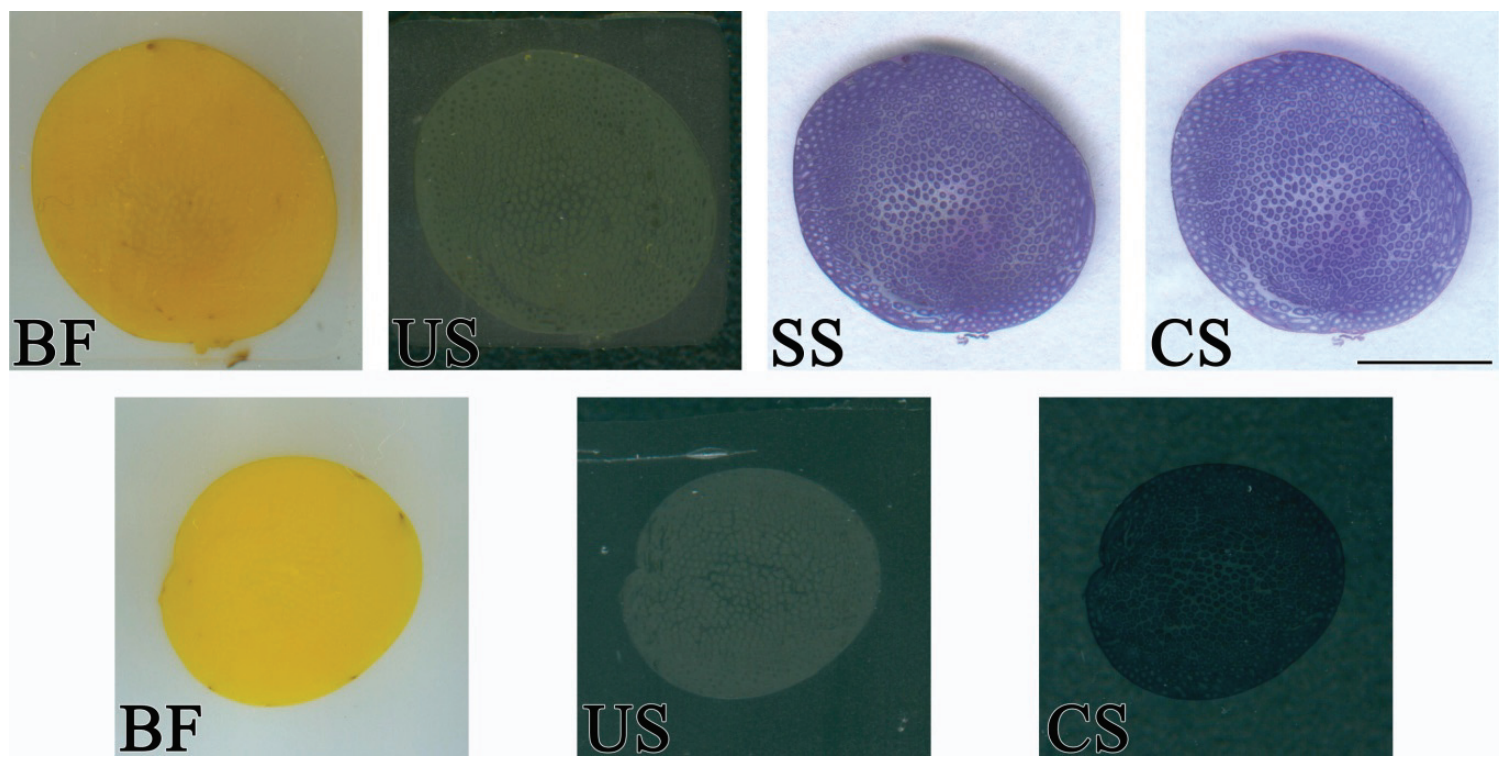

Fig. 1. Scanned images of the block face and sections, with the $Y$ axis representing the sectioning direction. Upper row (4 images) / lower row (3 images), from a tissue block of the First / Second Experiment. BF, the face of a paraffin embedded testicular tissue (adult rat) block left just after a section (US, unstained section on slide) was cut; the section was then stained (SS, stained section) and coverslipped (CS, coverslipped section). Section thickness set by the microtome: $10 \mu \mathrm{m}$ (upper row) and $5 \mu \mathrm{m}$ (lower row). Scale bar $=5 \mathrm{~mm}$. 
For measurement of actual section thickness and nuclear profile diameter, coverslipped section was observed on a computer screen (final magnification $\times$ 3010) using a $\times 100$ oil immersion lens (numerical aperture 1.30) of an Olympus BX53 microscope equipped with a stereology system (NewCAST, Visiopharm, Denmark). 14-23 (average 17.6) fields of view per section were sampled through the whole section in a systematic random manner by means of a computerassisted motorized stage (ProScan III, Prior Scientific Inc., USA). On each field 2 test frames (each $35 \mu \mathrm{m}$ $\times 26 \mu \mathrm{m}$ ) were superimposed. The left frame was first used to measure the section thickness: the upper surface of the section was first brought into focus within the frame area, and then the bottom surface focused; the distance between the 2 focal planes, which was measured by a microcator (Dr. Johannes Heidenhain GmbH, Germany) and shown on the screen, was a thickness of the section at the frame area (Peng et al., 2012; Xiang and Yang, 2014; Xiang et al., 2015; Guo et al., 2016). Both frames on each field were then used to sample and measure nuclear profiles: (i) the section was focused at $2 \mu \mathrm{m}$ below its upper surface, (ii) the nuclear profiles (in focus) of early round spermatids (Fig. 2), which are associated with late elongated spermatids in the wall of convoluted cylinder-shaped seminiferous tubules (Yang et al., 2004), were sampled according to the forbidden-line rule (Gundersen, 1977; Yang, 2012), and (iii) the vertical and horizontal diameters (Feret diameters) of each nuclear profile sampled were measured along the $\mathrm{Y}$ and $\mathrm{X}$ axes, respectively. (Before measurement of each section, the section image was rotated so that its $\mathrm{Y}$ axis on the screen represented the sectioning direction.) 20-34 (average 24.8) nuclear profiles were sampled and measured per section.

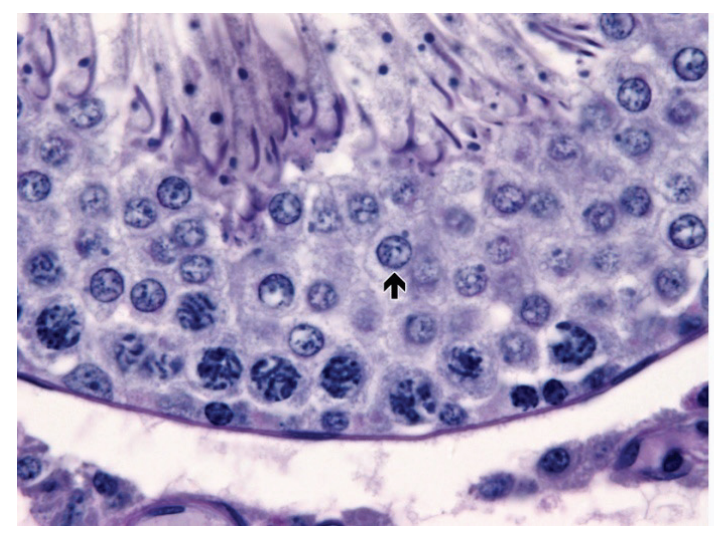

\section{SECOND EXPERIMENT}

In the First Experiment (above), (i) testicular tissue blocks were obtained from only 1 animal, and (ii) paraffin sections were first dried, immediately after mounting onto slides, flat on hotplate (i.e., the sectional plane parallel to the horizontal plane or worktable surface). This experiment was further carried out by (i) using tissue blocks from more animals, to address possible variation between animals, and (ii) drying newly mounted sections by vertically placing them (i.e., the sectional plane perpendicular to the horizontal plane), to address possible variation between methods of section drying which was shown to be important in section preparation (Guo et al., 2016). In brief, this Second Experiment was performed in the same way as in the First Experiment except the following protocols.

(i) Twelve testes were removed from 6 normal adult male Sprague-Dawley rats; one tissue block was obtained from each testis at an arbitrary (different) position along the testicular long axis.

(ii) Immediately after being floated onto slides from water bath $\left(37^{\circ} \mathrm{C}\right)$, paraffin sections were vertically placed (sectional plane perpendicular to horizontal plane) in a slide box, which was stored in an incubator $\left(37^{\circ} \mathrm{C}\right)$ for $24 \mathrm{hrs}$. To detect whether the drop-weight of water held under the section had an effect on the flattening of sections on slides, half (randomly chosen) of the 10 - or 5 - $\mu$ m-thick sections were placed with the sectioning direction (on the sectional plane) being perpendicular to the horizontal plane and the other half placed with the sectioning direction parallel to the horizontal plane.

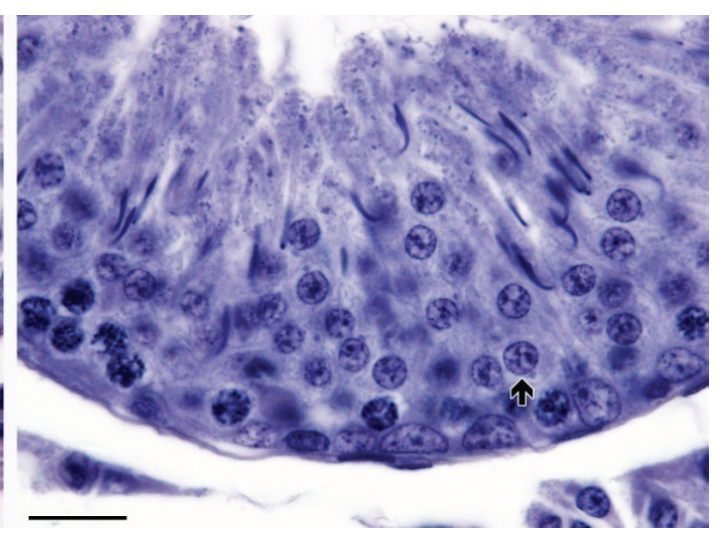

Fig. 2. Micrographs taken from 2 paraffin embedded testicular tissue (adult rat) sections. Left one: section thickness set at $5 \mu \mathrm{m}$, stained with periodic acid-Schiff's reagent and hematoxylin, First Experiment; right one: section thickness set at $10 \mu \mathrm{m}$, stained with hematoxylin alone, Second Experiment. The arrow ( $\boldsymbol{\uparrow}$ ) points to (i) a nuclear profile of round spermatids within the wall of the seminiferous tubule and (ii) the direction of sectioning. Scale bar $=20 \mu \mathrm{m}$. 
(iii) The SS was not scanned for measurement, since the section was not normally air-dried before coverslipping.

(iv) Sections were stained with hematoxylin alone, with-out the non-conventional periodic acidSchiff's reagent.

(v) For area estimation of scanned images, a smaller distance $(1 \mathrm{~mm})$ between the square-grid lines was chosen, as the section area was smaller.

(vi) For measurement of actual section thickness and nuclear profile diameter, 14-19 (average 16.7) fields of view and 14-31 (average 22.0) profiles of round spermatid nuclei were sampled per section.

\section{STATISTICS}

For the First or Second Experiment, comparison between the thicker $(10 \mu \mathrm{m})$ and thinner $(5 \mu \mathrm{m})$ section groups was made using the paired t-test; multiple comparisons were made between subgroups (BF, US, SS and CS) of either section group using the one way repeated measures analysis of variance in combination with the Student-Newman-Keuls method (Tables 13). The Student's $t$-test was used for comparison between some other groups and was indicated at respective places in the text. The statistical significance of difference was set at $P<0.05$.

Table 1. First Experiment: mean (coefficient of variation, i.e. standard deviation divided by the mean, in parenthesis) area and diameter of block faces and sections $(n=12)$. A section (thickness 5 or $10 \mu \mathrm{m}$ set by microtome) was cut from each of 12 paraffin-embedded testicular tissue (adult rat) blocks and then another section of different thickness (10 or $5 \mu \mathrm{m}$ ) cut from the same block. BF, the tissue block face left just after each section was cut; US, the unstained section on slide, not dewaxed; SS, the stained section, after dewaxing, staining, dehydration and clearing, not coverslipped; CS, the coverslipped section, after the mounting medium solidified. Vertical and horizontal diameters, Feret diameters measured along the sectioning direction and its perpendicular direction, respectively. ${ }^{\#} P \leq 0.05$ for comparison between the thicker and thinner section groups; ${ }^{*} \mathrm{P} \leq 0.05$ for comparison between the US and BF, SS and US, or CS and SS in either section group.

\begin{tabular}{cll}
\hline & $10 \mu \mathrm{m}$ sections & $5 \mu \mathrm{m} \mathrm{sections}$ \\
\hline Area $\left(\mathrm{mm}^{2}\right.$ ) of CSs (coverslipped sections) & $76.1(21.1 \%){ }^{*}$ & $76.8(21.0 \%)$ \\
Ratio of US to BF areas & $0.938(3.5 \%)^{*}$ & $0.935(2.5 \%)^{*}$ \\
Ratio of SS to US areas & $0.960(1.7 \%)^{*}$ & $0.959(2.4 \%)^{*}$ \\
Ratio of CS to SS areas & $1.029(4.0 \%)^{*}$ & $1.019(3.7 \%)$ \\
Vertical diameter (mm) of CSs ${ }^{*}$ & $9.48(12.8 \%)^{*}$ & $9.67(11.3 \%)$ \\
Ratio of US to BF vertical diameters & $0.928(1.2 \%)^{*}$ & $0.934(1.3 \%)^{*}$ \\
Ratio of SS to US vertical diameters & $0.977(1.3 \%)^{*}$ & $0.984(1.8 \%)^{*}$ \\
Ratio of CS to SS vertical diameters & $1.005(1.8 \%)$ & $1.010(1.6 \%)$ \\
Horizontal diameter (mm) of CSs & $10.20(10.3 \%)$ & $10.30(10.0 \%)$ \\
Ratio of US to BF horizontal diameters & $0.987(1.4 \%)^{*}$ & $0.994(1.0 \%)^{*}$ \\
Ratio of SS to US horizontal diameters & $0.992(0.9 \%)$ & $0.994(0.4 \%)^{*}$ \\
Ratio of CS to SS horizontal diameters & $1.004(0.8 \%)$ & $1.005(0.4 \%){ }^{*}$ \\
\hline
\end{tabular}

Table 2. Second Experiment: mean (coefficient of variation in parenthesis) area and diameter of block faces and sections $(n=12)$, obtained from another set of 12 paraffin-embedded testicular tissue (adult rat) blocks. * $P \leq 0.05$ for comparison between the thicker and thinner section groups; ${ }^{*} P \leq 0.05$ for comparison between the US and BF, or CS and US in either section group. See Table 1 for study design and abbreviations.

\begin{tabular}{lll}
\hline & $10 \mu \mathrm{m}$ sections & $5 \mu \mathrm{m}$ sections \\
\hline${\text { Area }\left(\mathrm{mm}^{2}\right) \text { of CSs (coverslipped sections) }{ }^{\#}}^{\text {Ratio of US to BF areas }}$ & $58.9(22.6 \%)$ & $56.9(23.1 \%)$ \\
Ratio of CS to US areas & $0.962(2.4 \%)^{*}$ & $0.947(3.1 \%)^{*}$ \\
Vertical diameter (mm) of CSs & $0.983(1.7 \%)^{*}$ & $0.980(2.3 \%)$ \\
$\quad$ Ratio of US to BF vertical diameters & $8.18(11.8 \%)$ & $8.16(11.9 \%)$ \\
Ratio of CS to US vertical diameters & $0.949(1.6 \%)^{*}$ & $0.944(2.3 \%)^{*}$ \\
Horizontal diameter (mm) of CSs & $0.993(1.1 \%)$ & $0.994(0.7 \%)$ \\
Ratio of US to BF horizontal diameters & $8.77(15.1 \%)$ & $8.73(14.7 \%)$ \\
Ratio of CS to US horizontal diameters & $0.997(0.4 \%)$ & $0.996(0.5 \%)^{*}$ \\
\end{tabular}


Table 3. Mean (coefficient of variation in parenthesis) actual section thickness and nuclear profile (round spermatids in the testis of adult rats) diameter $(n=12)$, measured with coverslipped sections. The vertical and horizontal Feret diameters of each nuclear profile were measured along the sectioning direction and its perpendicular direction, respectively. See Tables 1 \& 2 for study design.

\begin{tabular}{lll}
\hline & $10 \mu \mathrm{m}$ sections & $5 \mu \mathrm{m}$ sections \\
\hline First Experiment & $9.50(2.2 \%)$ & $4.85(9.2 \%)$ \\
$\quad$ Average of actual thicknesses of the section $(\mu \mathrm{m})$ & $6.65(1.9 \%)$ & $6.66(2.3 \%)$ \\
$\quad$ Average of vertical diameters of nuclear profiles $(\mu \mathrm{m})$ & $0.985(1.7 \%)$ & $0.977(1.4 \%)$ \\
$\quad$ & & \\
$\quad$ Average of ratios of vertical to horizontal diameters & $9.59(1.2 \%)$ & $4.78(2.2 \%)$ \\
Second Experiment & $6.77(1.8 \%)$ & $6.75(1.8 \%)$ \\
$\quad$ Average of actual thicknesses of the section $(\mu \mathrm{m})$ & $0.948(1.7 \%)$ & $0.943(1.2 \%)$ \\
$\quad$ Average of vertical diameters of nuclear profiles $(\mu \mathrm{m})$ & & \\
Average of ratios of vertical to horizontal diameters &
\end{tabular}

\section{RESULTS}

\section{AREA COMPRESSION}

In the First Experiment, the area of the CS was reduced by $7.3 \%-8.6 \%$ (in comparison with that of the BF) on average, with $74.7 \%-84.4 \%$ of the reduction (US in comparison with BF) being contributed by section compression in the process of section cutting, mounting and drying (Table 1 and Fig. 1). The vertical diameter of the CS was decreased by a comparable $7.2 \%-8.9 \%$, with $81.2 \%-91.4 \%$ of the decrease contributed by compression at the section acquiring procedure; in contrast, the decrease in the horizontal diameter, although significant, was small, only $0.7 \%$ 1.7\% (Table 1 and Fig. 1).

Staining after sectioning further decreased the section area (SS in comparison with US) by $4.0 \%-$ $4.1 \%$, while coverslipping appeared to increase it (CS in comparison with SS) by $1.9 \%-2.9 \%$ (Table 1 ).

In the Second Experiment, the CS area was reduced by $5.5 \%-7.2 \%$ (cf. BF), with its $69.5 \%-73.7 \%$ reduction contributed by the section acquiring induced compression (Table 2 and Fig. 1). [The average reduction of each CS's area (relative to its BF's area ) was $24.8 \%$ (for $10-\mu m$-thick sections) and $17.0 \%$ (5$\mu \mathrm{m}$-thick sections) smaller compared to the First Experiment, but statistical significance was not detected: the $P$ values of $t$-test used for comparison between the 2 experiments were larger than 0.20 .] The CS vertical diameter was decreased by a comparable $5.9 \%-$ $6.1 \%$, with its $87.8 \%-91.2 \%$ decrease contributed by section compression prior to staining, while the decrease in the horizontal diameter was only $0.8 \%-0.9 \%$ (Table 2).

\section{THICKNESS COMPRESSION}

In the First Experiment, the mean measured (actual) thickness of the CS was reduced (in comparison with the thickness set by the microtome) by $3.1 \%-5.0 \%$ on average and the within-section variation of thicknesses was $4.0 \% \pm 1.5 \%$ (mean \pm standard deviation, in the 10 - $\mu \mathrm{m}$-thick section group) and $6.8 \% \pm 1.8 \%(5-\mu \mathrm{m}$ thick section group) in terms of coefficients of variation (standard deviation divided by mean); similar reduction (by $4.1 \%-4.4 \%$ ) in the thickness occurred in the Second Experiment, but the coefficients (1.5\% $\pm 0.3 \%$ in the thicker section group and $2.3 \% \pm 0.5 \%$ in the thinner section group) of within-section thickness variation were considerably smaller (Table 3 ).

\section{COMPRESSION OF ROUND SPERMATID NUCLEI}

The vertical diameters of round spermatid nuclear profiles on the CS were reduced (in terms of reduction of the mean vertical to horizontal diameters ratio, relative to number 1 - the theoretical ratio for round particles) by $1.5 \%-2.3 \%$ (on average) in the First Experiment and $5.2 \%-5.7 \%$ in the Second Experiment (Table 3). The average reduction was 3.4-fold (for 10 - $\mu$ m-thick sections) and 2.4-fold (5- $\mu$ m-thick sections) larger in the Second Experiment compared to the First Experiment: the $P$ values of $t$-test used for comparison between the 2 experiments were smaller than 0.001. However, apparent nuclear compression along the sectioning direction was hardly appreciable, if not measured, on sections from both experiments (Fig. 2).

\section{COMPARISON BETWEEN THICKER AND THINNER SECTION GROUPS}

There were not significant differences between the thicker $(10 \mu \mathrm{m})$ and thinner $(5 \mu \mathrm{m})$ section groups in all parameters estimated except that the thicker section group was associated with (i) a 3.4\%-3.7\% larger area of the CS or US (cf. the area in the thinner section group) in the Second Experiment, (ii) a 1.6\%-2.1\% smaller vertical diameter of the CS or SS in the First 
Experiment, and (iii) a 31.5\%-37.5\% smaller coefficients of within-section thickness variation in both Experiments (Tables 1-3).

\section{DROP-WEIGHT EFFECT OF WATER UNDER SECTION IN DRYING}

To determine whether different orientations of vertically placed sections in drying had different drop-weight effects on section area and thickness compression, the 12 thicker $(10 \mu \mathrm{m})$ or thinner $(5 \mu \mathrm{m})$ sections in the Second Experiment were divided into two subgroups, in which the sections (6 each subgroup) were placed with the sectioning direction being perpendicular (1 subgroup) or parallel (the other subgroup) to the horizontal plane. Comparison using the $t$-test showed that there were not significant differences $(P>0.10)$ between the 2 subgroups in all parameters measured. That is, no drop-weight effects were found; therefore, results of the 2 subgroups were not separately reported.

\section{DISCUSSION}

Compression of sections involves compressions of both section area (dimensions) and section thickness. With the use of microcator - best choice for section thickness measurement (Dorph-Petersen et al., 2001), thickness compression of paraffin sections (for soft biological tissues) was found to be from a few percent to $30 \%$ by some studies (Gardella et al., 2003; Bas et al., 2009; Yang, 2012; Peng et al., 2012; Xiang et al., 2015; Guo et al., 2016). For example, using the same Leica paraffin, microtome and blades and the same thickness measuring method, we previously showed that the thickness compression of the rat lung, heart, liver, testis and spinal cord tissues was $16 \%-28 \%$, and thicker or thinner sections (thickness set at 5, 10, 20, 30 or $40 \mu \mathrm{m}$ ) did not necessarily mean a larger or smaller compression (Peng et al., 2012). The current study showed, however, the compression was only $3 \%-5 \%$. Considering that heating paraffin sections at $90^{\circ}-140^{\circ} \mathrm{C}$ reduced the thickness by half (Xiang et al., 2015) and drying paraffin sections at $54^{\circ} \mathrm{C}$ reduced the thickness by 15\% (Guo et al., 2016), we speculate that the variation of thickness compression might be largely attributed to the duration and temperature of section heating or drying, which might be quite different between studies. Temperature of water bath might be another contributor as we also showed that the compression (by $8.4 \% \pm 3.6 \%$ ) of $10 \mu \mathrm{m}$ thick paraffin sections was significantly higher when the water bath temperature was higher, $49^{\circ} \mathrm{C}$ (unpublished study).

Few researchers studied the area compression of sections although it seemed easier to measure area than thickness. The present study found a 5\%-9\% area compression of 5-10 $\mu \mathrm{m}$ thick paraffin sections. Similarly, one of our unpublished studies (above) demonstrated an $8 \%$ area compression. In stark contrast, we previously found a $30 \%$ area compression in 2 separate studies with 7-8 $\mu$ m thick paraffin embedded testicular tissue (mice) sections (Yang and Cui, 1989; Li et al., 1990). Much of this discrepancy might be ascribed to the previously used paraffin and knife and area estimation methods.

The 5\%-9\% area compression shown in the present study was primarily the result of section compression along the sectioning direction as a similar degree (6\%-9\%) of linear (vertical) compression in the direction was found, with the linear horizontal compression being only approximately $1 \%-2 \%$. It is mechanically reasonable that the vertical compression was associated with a degree of horizontal compression. This is different from the study of Boonstra et al. (1983) which showed 2.2\%-4.3\% vertical compression and $0.4 \%-3.0 \%$ horizontal expansion of paraffin embedded cervical tissue sections. But the latter study, without reporting the section thickness or measuring instrument, measured distances between the tissue "landmarks" that could be deformed after sectioning, thus misrepresenting the sectioning direction.

The area compression estimated by comparison between dimensions of the unstained section and the block face was the overall effect of the processes of section cutting, mounting and drying. As it was unlikely that the section would be compressed, if not expanded, during the mounting and drying process, the area compression must have been primarily the result of the cutting procedure per se. That is, it was the blade edge and the cutting stroke or force that compressed the section. Results obtained at later processes of section preparation demonstrated that the overall effect of the following staining (including dewaxing, dehydration and clearing) and coverslipping procedures was a further compression, though to a lesser extent (accounting for $15.6 \%-30.5 \%$ of the total area compression). This suggested that the paraffin sections mounted and dried on slides were fully flattened (see also Guo et al., 2016), or further flattening (if present) of sections or structures after mounting and drying was limited.

The spermatid nuclei sampled for measurement in this study are round, spherical (Yang et al., 2004). Even if they are slightly elliptical, they may well be assumed to be isotropic in general considering their distribution in the wall of convoluted cylindrical seminiferous tubules. Therefore smaller vertical diameter of the nuclear profiles, as shown in the present study 
(Table 2), signified a vertical compression of the nuclei, consistent with the vertical compression of sections. But the degree of the vertical nuclear compression (by 1.5\%-2.3\%) shown in the First Experiment was much smaller than that of the vertical section compression (by $7.2 \%-8.9 \%$ ). It was also noted that the (round) nuclei of spermatocytes and early round spermatids were not markedly compressed even with a $30 \%$ area compression of the sections (Yang and Cui, 1989). These indicated a considerable differential or non-uniform compression: different structures within sections have different degrees of compression. The implications were obvious: (i) nuclei in soft tissues, the key structures identified by conventional microscopy, might be "harder" structures (compared with their surrounding cytoplasm and intercellular structures) resistant to compression; (ii) the method of using spherical structures to estimate section compression (Weibel, 1979) was not applicable.

Interestingly, a much larger (vertical) compression (by $5.2 \%-5.7 \%$ ) of the nuclear profiles was found in the Second Experiment, the degree of compression being close to that of the section compression (above). The smaller nuclear compression in the First Experiment may be explained by the slower "horizontal" drying of sections (i.e. sections placed flat immediately after mounting from water bath), which allowed tissue structures more time for flattening in water. That is, although faster "vertical" drying of sections performed in the Second Experiment (i.e. sections vertically placed immediately after mounting) may be good for prevention of section detachment, deformation or destruction (Guo et al., 2016), it may not be good for further flattening of structures within paraffin sections.

Apart from the above marked difference between the 2 experiments, it seemed that different methods of section drying used in the experiments did not have considerably or significantly different effects on the parameters measured in this study.

The present study also demonstrated that different thicknesses ( 5 and $10 \mu \mathrm{m}$ ) did not have considerably different effects on section area and thickness compression. It may be worth mentioning, however, that both experiments showed a consistent larger within-section variation of thickness with the thinner $(5 \mu \mathrm{m})$ sections. This might imply that thinner (e.g. $1-3 \mu \mathrm{m})$ sections might have even larger within-section thickness variation, which might affect the microscopic observation or quality of the sections.

Comparison between the block face and the section was based on assumptions that (i) they should be the same in terms of shape and size if no changes had occurred in the cutting, staining or coverslipping of the section, and (ii) no peripheral parts of the section were missing during the section preparation and the boundary of the block face and section could be unambiguously identified. The assumptions were reasonably true in the current study in that (i) the size and shape of the block face should be essentially the same as that of the unstained section (if no changes had occurred in the processing) as the section thickness was only about one thousandth of the ellipsoidal testicular diameters. Even if it might not be so, there was equal probability that the section was smaller or larger, very slightly if so, than the block face. (ii) A thick capsule (tunica albuginea) encircles the whole tissue section, making the section boundary clearly observable (Fig. 1).

It may be asked whether the thickness and refraction of the coverslip and balsam affected the scanned size of the coverslipped section. The answer is no according to our pilot study, in which we used 4 smaller pieces of glass coverslip (painted with a blue nail enamel) as stained "sections" and compared the images scanned before and after the "sections" were coverslipped (unpublished data). As to the accuracy of the section thickness set by the microtome, we showed, in a separate study using a digital caliper (Shanghai Meinaite Industrial Company Ltd., China; accuracy $10 \mu \mathrm{m})$ to measure a few methacrylate embedded testicular tissue blocks before and after serial sectioning, that the thickness of $25 \mu \mathrm{m}$ set by the same microtome was estimated to be $24.2 \mu \mathrm{m}$ on average (unpublished study). This serves as a justification that the thickness setting of the microtome should be acceptable in the present study. [However, if we preferred to believe the accuracy of the indirect manual measurement with the aid of the digital caliper (measurement range $10 \mu \mathrm{m}-150 \mathrm{~mm}$ ), rather than the accuracy of the microtome (section thickness setting range 1-60 $\mu \mathrm{m}$ ), the accuracy checking measurement would suggest a considerably thinner (by an average of 3.2\%) actual thickness of the sections cut with the microtome. That is, the overall thickness compression of the sections during processing would be largely negligible in the present study.]

In summary, the present study reported the area compression (by 5.5\%-8.6\%), thickness compression (by 3.1\%-5.0\%) and within-section coefficient of thickness variation $(4.0 \%-6.8 \%)$ of 4 sets of 12 paraffin embedded testicular sections (thickness 5-10 $\mu \mathrm{m}$ ). And an effect (in terms of morphometry) of the compression on structures was found: a $1.5 \%-2.3 \%$ compression of the vertical diameters of round spermatid 
nuclear profiles in 2 sets of sections and a $5.2 \%-5.7 \%$ compression in the other 2 sets of sections, indicating a non-uniform compression of structures within some sections depending on procedures of section drying. It should be pointed out, however, that (i) the effects of section compression alone on non-spherical structures within sections could hardly be estimated because of the lack of "control" (comparable sections or sectional images without the section compression only) or "standard" (results from "control"). (ii) And the present study dealt with only one type of soft tissue and some procedures of tissue processing. More studies are needed to demonstrate variation of the results between tissues and/or procedures. Probably, such physical or mechanical results are more affected by quality of the embedding medium and sectioning device (above) than types of tissue or procedures of processing. (iii) Furthermore, the present study did not deal with tissue swelling or, more likely overall, shrinkage associated with such tissue processing as fixation, dehydration and embedding, which might affect morphometry to a greater degree. While the combined or total effects of tissue shrinkage and section compression of paraffin sections could be evaluated by comparing results obtained with glycol methacrylate embedded sections (e.g. Zhao et al., 2010), it is always advisable, if staining, time or condition permitted, to use resin sections with much less tissue shrinkage and section compression for morphometric studies (Yang, 2012).

\section{ACKNOWLEDGMENTS}

This study was supported by grants from the Research Development Program of North Sichuan Medical College (CBY13-A-QN35 \& CBY13-A-ZP05).

\section{REFERENCES}

Bas O, Odaci E, Kaplan S, Acer N, Ucok K, Colakoglu S (2009). $900 \mathrm{MHz}$ electromagnetic field exposure affects qualitative and quantitative features of hippocampal pyramidal cells in the adult female rat. Brain Res 1265:178-85.

Boonstra H, Oosterhuis JW, Oosterhuis AM, Fleuren GJ (1983). Cervical tissue shrinkage by formaldehyde fixation, paraffin wax embedding, section cutting and mounting. Virchows Arch A Pathol Anat Histopathol 402:195-201.
Dorph-Petersen KA, Nyengaard JR, Gundersen HJ (2001). Tissue shrinkage and unbiased stereological estimation of particle number and size. J Microsc 204:232-46.

Gardella D, Hatton WJ, Rind HB, Rosen GD, von Bartheld CS (2003). Differential tissue shrinkage and compression in the z-axis: implications for optical disector counting in vibratome-, plastic- and cryosections. J Neurosci Methods 124:45-59.

Gundersen HJG (1977). Notes on the estimation of the numerical density of arbitrary profiles: the edge effect. J Microsc 111:219-23.

Guo Y, Xiang Y, Yang ZW (2016). Drying paraffin sections on hotplate unadvisable. J Histol Histopathol 3:4.

Li H, Yang ZW, Cui CH, Zhang ZF (1990). Comparison of resin and paraffin sections in a quantitative study. Journal of North Sichuan Medical College 5(4):48-50. [Article in Chinese]

Peng B, Chen MS, Li H, Yang ZW (2012). Comparison of the compressions of paraffin-embedded sections from different organs. Chinese J Stereol and Image Anal 17: 219-5. [Article in Chinese]

Weibel ER (1979). Stereological Methods. Vol. 1 Practical Methods for Biological Morphometry. London: Academic Press. 150-2.

Xiang Y, Guo Y, Huang XL, Peng B, Yang ZW (2015). Effects of heating on the adhesion, thickness and staining of paraffin sections. Chinese Journal of Stereology and Image Analysis 20:52-7. [Article in Chinese]

Xiang Y, Yang ZW (2014). Detachment of methacrylateembedded sections from microscope slides can be prevented by heating on hotplate. J Histol Histopathol 1:10.

Yang ZW (2012). Essential Tools for Morphometric Studies of Biological Tissues: Practical Stereological Methods. Beijing: Science Press. [Book in Chinese]

Yang ZW, Cui CH (1989). Tissue shrinkage and section compression of paraffin sections of testicular tissue. Journal of North Sichuan Medical College 4(4):4-7. [Article in Chinese]

Yang ZW, Guo Y, Lin L, Wang XH, Tong JS, Zhang GY (2004). Quantitative (stereological) study of incomplete spermatogenic suppression induced by testosterone undecanoate injection in rats. Asian J Androl 6:291-7.

Zhao YY, Wang YP, Guo Y, Zhang RD, Yang ZW (2010). Effects of magnification and embedding medium on a stereological study of testicular structures. Chinese Journal of Stereology and Image Analysis 15:191-4. [Article in Chinese] 